\title{
The Effect of Proton Pump Inhibitors on Glycemic Control in Patients with Type 2 Diabetes
}

\author{
Kelly Villegas, PharmD, ${ }^{1}$ Joy L. Meier, PharmD, BCACP, ${ }^{1}$ Maureen Long, PharmD, \\ Julio Lopez, PharmD, ${ }^{1}$ and Arthur Swislocki, MD, FACP ${ }^{2,3}$
}

\begin{abstract}
Background: Type 2 diabetes patients have decreased pancreatic beta cell mass with a decline in beta cell function. Gastrin has increased beta cell proliferation in vitro and in animal studies. High gastric acid levels inhibit gastrin secretion. Proton pump inhibitors (PPIs) lower gastric acid, subsequently increasing gastrin levels. This may stimulate beta cell proliferation and function, and improve glycemic control. Studies with small numbers of type 2 diabetes patients have shown a slightly lower A1C in those taking PPI versus non-PPI users. Methods: This study was a retrospective multicenter electronic data analysis using data obtained from health care facilities within Veterans Integrated Service Network (VISN) 21. Patients were included if they had established care within VISN 21 and had type 2 diabetes with an $\mathrm{A} 1 \mathrm{C}>6.5 \%$, were started on a PPI concurrently with stable doses of metformin or sulfonylurea (SFU) monotherapy, had at least two documented A1C values, and had a medication possession ratio $>80 \%$ for metformin, SFU, or a PPI. Veterans were excluded if they were using insulin, combination antihyperglycemic therapy, or oral corticosteroids. A control group not using PPI was also identified.

Results: There was a statistically significant decrease in A1C within each group. However, there was no statistically significant difference between the PPI and control group in the post-A1C.

Conclusion: In patients with type 2 diabetes, A1C improved in both groups, but PPI addition did not affect glycemic control. Future randomized controlled trials are needed to determine the value of PPIs as a treatment option for patients with type 2 diabetes.
\end{abstract}

Keywords: diabetes, proton pump inhibitors, glycemic control

\section{Background}

Patients with type 2 diabetes have decreased panP creatic beta cell mass with an eventual decline in beta cell function. ${ }^{1,2}$ Gastrin is a hormone that has been shown to increase beta cell proliferation in vitro and in animal studies. $^{2-6}$ Due to a negative feedback loop between gastrin and gastric acid, gastrin secretion is inhibited by high gastric acid levels. ${ }^{5}$ Because proton pump inhibitors (PPIs) lower gastric acid, subsequently increasing gastrin levels, it is theorized that this may stimulate beta cell proliferation and function and improve glycemic control. Six recent studies with small numbers of subjects with type 2 diabetes have shown a slightly lower A1C in those subjects taking a PPI when compared to those not taking a PPI. ${ }^{2-4,7-9}$

The purpose of this study was to replicate previous studies and evaluate the effect of PPIs on glycemic control in pa- tients with type 2 diabetes by comparing A1C values to a similar group of patients with type 2 diabetes not using a PPI.

\section{Methods \\ Study design}

This study was approved by the Institutional Review Board of the Veterans Affairs (VA) Northern California Health Care System. The study design was a retrospective multicenter electronic data analysis using data obtained from health care facilities within Veterans Integrated Service Network (VISN) 21, which includes VA facilities in Northern Nevada, Central and Northern California, Hawaii, and The Philippines. Demographic, diagnostic, laboratory, and prescription data were organized and analyzed using Microsoft SQL tables. Hbalc and routine chemistry studies were performed in each VA

\footnotetext{
${ }^{1}$ Pharmacy Service and ${ }^{2}$ Medical Service, VA Northern California Health Care System, Martinez, California

${ }^{3}$ Department of Internal Medicine, UC Davis School of Medicine, Sacramento, California.
} 
TABle 1. Baseline Demographics

\begin{tabular}{lccc}
\hline & PPI & No PPI & P \\
\hline Male $(n, \%)$ & $187(96)$ & $2244(96)$ & 0.85 \\
Age (years, SD) & $67.4(10.5)$ & $66.7(10.9)$ & 0.44 \\
A1C, baseline (\%, SD) & $7.3(0.7)$ & $7.3(0.9)$ & 0.14 \\
K+, baseline (mEq/L, SD) & $4.2(0.4)$ & $4.2(0.4)$ & 0.35 \\
BMI, baseline (kg/m 2 , SD) & $31.0(6.1)$ & $31.3(6.2)$ & 0.43 \\
\hline
\end{tabular}

BMI, body mass index; PPI, proton pump inhibitor.

station's clinical laboratory; HbA1c assays are standardized by the National Glycohemoglobin Standardization Project. The data collected were used to determine baseline characteristics and medication adherence.

\section{Patients}

VA patients were included in the analysis if they had a PPI prescription written at any station within VISN 21 between January 1, 2005, and December 31, 2011, and carried a diagnosis of type 2 diabetes, which the authors defined as having at least two outpatient or hospital encounters at any VISN 21 station coded with an ICD9 code for diabetes within the two years before PPI initiation. Veterans were also required to have established care within VISN 21, which the authors defined as having outpatient visits or medication fills documented for at least 3 months, but no more than a year before PPI initiation. To assess diabetic control, a baseline $\mathrm{A} 1 \mathrm{C}>6.5 \%$ was required along with at least two A1C values, one within 365 days before PPI initiation and one at least 90, but no greater than 365 days following PPI initiation. PPI had to be taken concurrently with stable doses of metformin or sulfonylurea (SFU) monotherapy, and more than one prescription fill was necessary for metformin, SFU, and/or PPI. Veterans were excluded if they were using insulin or combination antihyperglycemic therapy, or oral corticosteroid use documented within 90 days of the baseline and follow-up A1C. The Veterans in the control group were Veterans with type 2 diabetes, who received care during the same timeframe and met the same criteria, but had not received PPI.

\section{Adherence assessment}

Adherence was assessed using the medication possession ratio (MPR). The MPR was calculated as the sum of days supply for all fills over a specific period of time divided by the number of days elapsed during the period. ${ }^{10}$ Only Veterans with an MPR $>80 \%$ for metformin, SFU, or PPI, considered to be adequate adherence, were included in the analysis.

\section{Primary objective}

PPI effect on glycemic control was evaluated by comparing the $\mathrm{A} 1 \mathrm{C}$ before and after PPI initiation to the $\mathrm{A} 1 \mathrm{C}$ in a group of patients with type 2 diabetes not taking a PPI. Patients were required to be on a PPI for at least 90 days to determine the effect on A1C. We identified the pre-A1C in the period between the PPI start date and 365 days prior; if more than one $\mathrm{A} 1 \mathrm{C}$ was documented in the pre-PPI period, we pulled the A1C closest to the PPI start date. If there was more than one $\mathrm{A} 1 \mathrm{C}$ documented in the post-A1C analysis period, from 90 to 365 days out, we chose the $\mathrm{A} 1 \mathrm{C}$ closest to the 90-day point. To ensure that patients were on PPI therapy at the time of post-A1c, we looked for a PPI fill after the post-A1C and identified the first fill after the post-A1C as the last PPI fill. We looked for initiation of metformin or SFU monotherapy between the PPI start date and 6 months prior. We looked for the last metformin or SFU fill date between the last PPI fill and 6 months after. If there was more than one fill documented in this time frame, we took the fill closest to the last PPI fill date.

\section{Secondary objective}

Differences between the treatment and control groups in factors known to affect glycemic control were also assessed, which were use of an atypical antipsychotic, niacin, statin, or thiazide diuretic, body mass index (BMI) changes, and serum potassium levels. The authors considered patients as taking an atypical antipsychotic, niacin, statin, or thiazide diuretic, drugs known to affect glucose control, by looking for a dispense date for any of these medications between 90 and 185 days before the pre-A1C, as well as a prescription fill date up to 180 days after the post-A1C to determine that therapy continued throughout the treatment analysis period.

\section{Statistical analysis}

To ensure a sample size adequate for the evaluation of an effect on $\mathrm{HbAlc}$, a power analysis was performed assuming a two-tailed test, alpha of 0.05 , beta of 0.2 , effect size for A1C

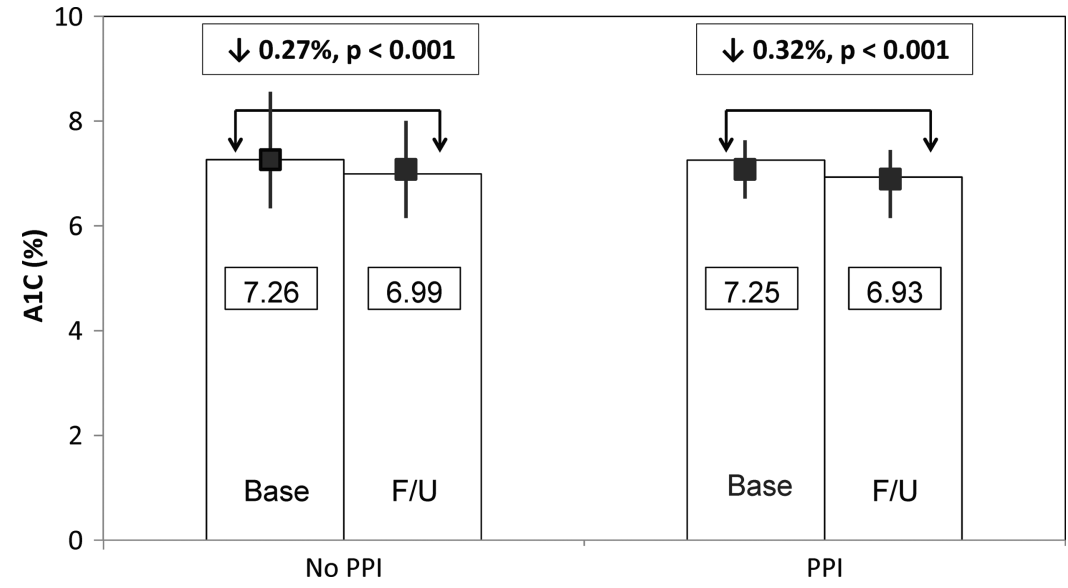

FIG. 1. Results: average A1C. The data show a statistically significant decrease in A1C within each group. However, when we compared the post-A1C in the control and treatment group, there was no statistically significant difference between the treatment and control group. PPI, proton pump inhibitor. 
FIG. 2. Results: BMI. There were no significant differences in pre-BMI and postBMI in the treatment and control groups. BMI, body mass index.

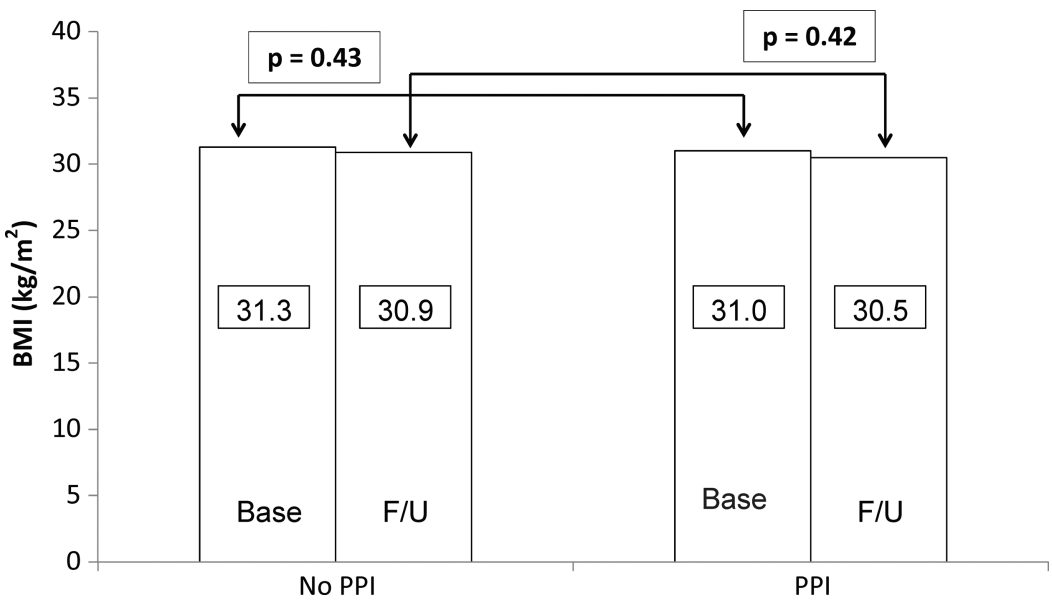

of 0.5 , and standard deviation of 1.49 . We determined that a sample size of 59 was needed to have adequate power to show a difference between the treatment and control group.

Independent group $t$-test and Mann-Whitney Rank sum test were used to analyze the difference in baseline and follow-up A1C, potassium, eGFR, and BMI between the treatment and control groups. The paired $t$-test and Wilcoxon Signed-Rank test were used to analyze the baseline and follow-up A1C within each group. The chi-squared test was used to compare the difference in use of medications known to affect glycemic control between the two groups. Statistical analysis was performed with SigmaPlot, version 12.5 (Systat Software, San Jose, CA)

\section{Results}

\section{Patient characteristics}

A total of 195 patients were in the treatment group and 2330 patients were in the control group for the final analysis. There were no significant differences in baseline characteristics between the treatment and control groups (Table 1).

\section{PPI effect on $\mathrm{HgbA1C}$}

There was a statistically significant decrease in A1C within each group (control and PPI treated). However, there was no statistically significant difference between the treatment and control group in the post-A1C (Fig. 1).

\section{Results of secondary objective analysis}

There were no significant differences in pre-BMI and post-BMI (Fig. 2), serum potassium (Fig. 3), or use of other medications known to affect glycemic control (Fig. 4) between the treatment and control groups. Doses of oral hypoglycemic agents were constant in both the treatment and control groups. Renal function remained essentially constant as well (Table 2).

\section{Discussion}

Our study demonstrates that there was a slight A1C decrease in both groups, but there was no significant difference in follow-up A1C between the treatment and control group. The analysis was adequately powered to show a difference between the study groups, as we only needed a sample size of 59 in each group. There were no significant differences between the study groups in factors known to affect glycemic control during the treatment period, such as weight, ${ }^{11}$ potassium, ${ }^{12}$ and adherence. ${ }^{13,14}$ Dietary intake was consistent, as shown by stable BMI, and not likely to have changed glycemic content. ${ }^{15}$ Both groups achieved excellent glycemic control as demonstrated by A1C values. ${ }^{16}$
FIG. 3. Results: potassium. There were 1031 patients missing prepotassium values and 976 missing postpotassium values. However, there were significant differences in prepotassium and postpotassium levels between the treatment and control groups.

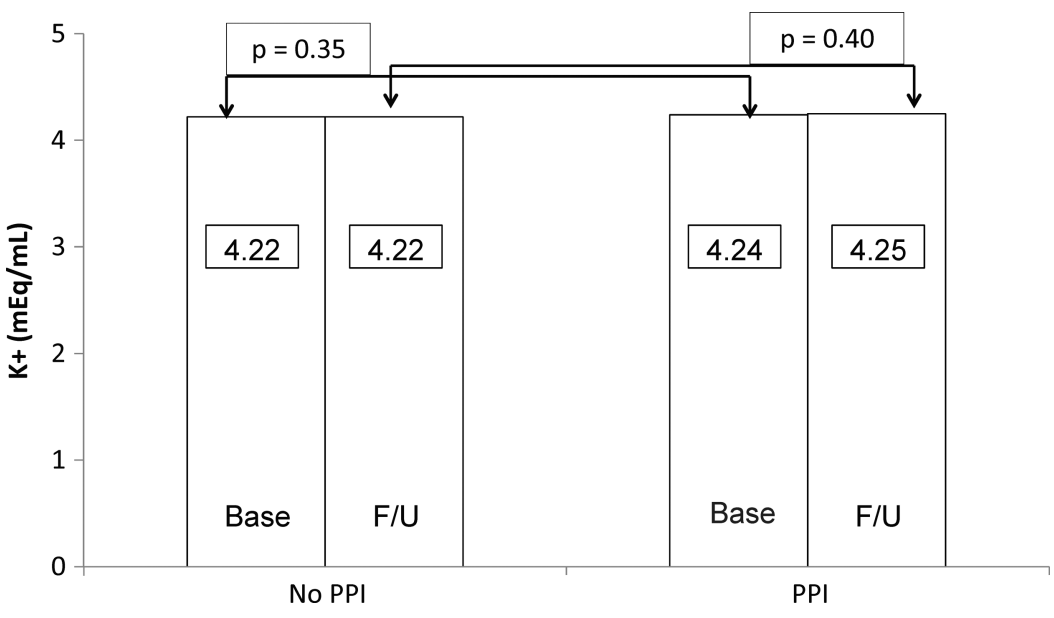




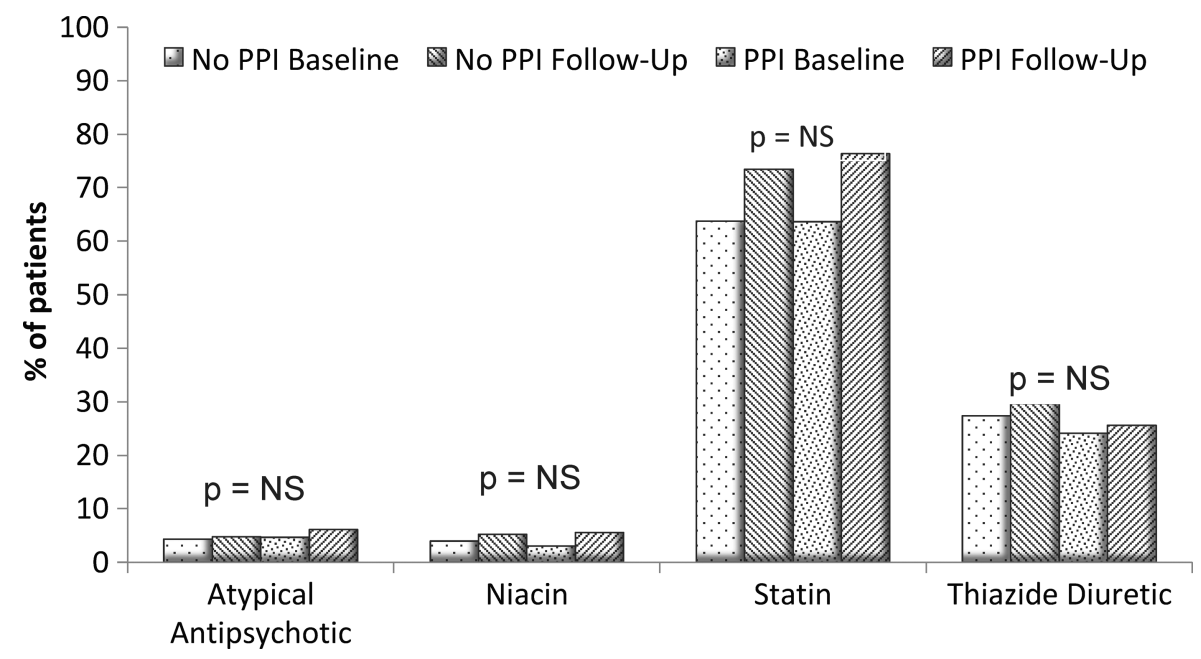

FIG. 4. Results: atypical antipsychotics, niacin, statins, and thiazide diuretics. There were no significant differences in use of atypical antipsychotic, niacin, statins, or thiazide diuretics at baseline and at follow-up in the treatment and control groups. Know the range of the $P$-values and how close they get to 0.05 . NS, not significant.

Recent reports have documented a potential increased risk of renal failure in chronic PPI users. ${ }^{17}$ In our population, renal function remained constant. Furthermore, impact of renal function on our primary outcome variable, $\mathrm{HbA1c}$, is modest, particularly since renal function was largely unchanged over the study period. ${ }^{18}$

Two recent reviews have supported the overall lack of effect of PPI on glycemic control. Takebayashi and Inukai also considered the reported positive effect of gastrin on pancreatic $\beta$-cell replication. In their literature review, they opine that, in general, PPI agents have benefit in terms of glycemic control, although this is not a universal finding. This benefit appears to be independent of the type of PPI. In addition, the glycemic benefit appears to be dependent on basal $\mathrm{HbA1c}$ in that the greatest benefit is present with higher baseline HbA1c values. The mechanisms of this apparent benefit are unclear; it is unlikely that there is, in humans, $\beta$ cell neogenesis like there is in rodents. There may be effects on postprandial insulin secretion, downregulation of ghrelin, and other possible effects, including a possible enhancement of the effect of endogenous GLP-1 on $\beta$-cell neogenesis. ${ }^{19}$

Another recent review, by Gómez-Izquierdo and $\mathrm{Yu}$, employed a focused literature review and met-analysis to address the same question. In contrast to the analysis of Takebayashi and Inukai, these authors reported that, while there was no consistent effects of PPI on HbA1c, pantoprazole therapy did show glycemic benefit. ${ }^{20}$ These discrepant results, based on literature review, are testimony to what may be at most a modest and inconsistent effect of PPI on glycemic control, dependent on study design and patient selection.

\section{Limitations}

Because this study is a retrospective analysis, it only demonstrates an association between PPI use and effect on A1C, and does not establish a causal relationship between the two variables. Over-the-counter (OTC) PPIs are generally expensive and Veterans get their medications for free or a nominal co-pay, so the inability to determine OTC PPI use is probably insignificant. We were limited to electronic data for this analysis. The data are only as accurate and reliable as the documentation. This study population was primarily male veterans with type 2 diabetes, and therefore it is difficult to determine the generalizability to nonveterans with uncontrolled or type 1 diabetes. In addition, many patients receiving PPI take them for relatively short courses, or intermittently, and our inclusion criterion of use for $\geq 90$ days would not have included these individuals. It is also possible

Table 2. Changes Over Study Period

\begin{tabular}{|c|c|c|c|c|c|c|}
\hline \multirow{2}{*}{$\begin{array}{l}\text { Parameter } \\
\text { Drug class }\end{array}$} & \multicolumn{3}{|c|}{ PPI group } & \multicolumn{3}{|c|}{ No-PPI group } \\
\hline & Pre & Post & $\mathrm{P}$ & Pre & Post & $\mathrm{P}$ \\
\hline \multicolumn{7}{|c|}{ Sulfonylurea (mg/day) } \\
\hline Glipizide & $10.3 \pm 6.5$ & $10.3 \pm 6.5$ & NS & $10.6 \pm 9$ & $10.6 \pm 9$ & NS \\
\hline Glyburide & $7.8 \pm 4.9$ & $7.8 \pm 4.9$ & NS & $8.7 \pm 6.4$ & $8.7 \pm 6.4$ & NS \\
\hline Tolazamide & a & & & $544.64 \pm 292.56$ & $544.64 \pm 292.56$ & NS \\
\hline Tolbutamide & $250^{\mathrm{b}}$ & 250 & NS & $2481.5 \pm 1198.4$ & $2481.5 \pm 1198.4$ & NS \\
\hline \multicolumn{7}{|c|}{ Biguanide (mg/day) } \\
\hline Metformin & $1481.7 \pm 541.7$ & $1481.7 \pm 541.7$ & NS & $1377.8 \pm 577$ & $1377.8 \pm 577$ & NS \\
\hline \multicolumn{7}{|c|}{ Renal function (mL/min) } \\
\hline
\end{tabular}

Data expressed as mean $\pm \mathrm{SD}$.

${ }^{a}$ No patients in this category.

bnly one patient in this category.

NS, not significant. 
that the glycemic response to PPI might be different with other oral hypoglycemics that may have different effects on the GI tract, for example, DPP-4 agents, alpha-glucosidase inhibitors, or SGLT-2 inhibitors.

\section{Conclusion}

We showed reasonable glycemic control in a cohort of veterans with diabetes. Addition of a PPI to their regimen did not affect their glycemic control. This is in contrast to prior recent studies. Even though this was a retrospective analysis, we applied stricter control criteria and had a larger study population. We looked at factors known to affect glycemic control (BMI changes, potassium changes, and other medications) and determined there was no difference between the control and treatment groups with respect to these parameters; therefore, we can be more confident in our results. Our study showed that PPIs do not have a significant effect on glycemic control, indicating they do not put patients at risk for hypoglycemia. Indeed, there is some evidence that the combination of PPI and metformin may exacerbate the risk of either agent to provoke B12 deficiency. ${ }^{21}$ Future prospective randomized controlled trials are still needed to verify this finding. Improved glycemic control is likely related to improvement in secular factors affecting our diabetic population as a whole.

\section{Acknowledgments}

This material is the result of work supported with resources and the use of facilities at the VA Northern California Health Care System. The contents do not represent the views of the U.S. Department of Veterans Affairs or the United States Government. Preliminary data have been presented at the 2012 Western States Conference, Asilomar, CA, May 24, 2012; the 2012 California Northstate University College of Pharmacy Residency Symposium, Sacramento, CA, May 7, 2012; the Western Section meeting, AFMR, Carmel, CA, February 2013; and the 95th Annual Meeting of the Endocrine Society, San Francisco, CA, June 2013.

\section{Author Disclosure Statement}

The authors report no commercial associations that might create a conflict of interests, actual or potential.

\section{References}

1. Butler AE, Janson J, Bonner-Weir S, et al. B-cell deficit and increased b-cell apoptosis in humans with type 2 diabetes. Diabetes 2003;52:102-110.

2. Hove KD, Færch K, Bödvarsdóttir TB, et al. Treatment with a proton pump inhibitor improves glycaemic control in type 2 diabetes patients-A retrospective analysis. Diabetes Res Clin Pract 2010;90:72-74.

3. Bodvarsdottir TB, Hove KD, Gotfredsen CF, et al. Treatment with a proton pump inhibitor improves glycaemic control in psammomys obesus, a model of type 2 diabetes. Diabetologia 2010;53:2220-2223.

4. Mefford IN, Wade EU. Proton pump inhibitors as a treatment method for type II diabetes. Med Hypotheses 2009; 73: 29-32.
5. Wolfe MM, Soli AH. The physiology of gastric acid secretion. N Engl J Med 1988;319:1707-1715.

6. Rooman I, Lardon J, Bouwens L. Gastrin stimulates B-cell neogenesis and increases islet mass from transdifferentiated but not from normal exocrine pancreas. Diabetes 2002;51: 686-690.

7. Crouch MA, Mefford IN, Wade EU. Proton pump inhibitor therapy associated with lower glycosylated hemoglobin levels in type 2 diabetes. J Am Board Fam Med 2012;25: 50-54.

8. Boj-Carcellar D, Bocos-Terraz P, Moreno-Vernis M, et al. Are proton pump inhibitors a new antidiabetic drug? A cross sectional study. World J Diabetes 2011;2:217-220.

9. Mefford IN, Mefford JT, Burris CA. Improved diabetes control and pancreatic function in a type 2 diabetic after omeprazole administration. Case Rep Endocrinol 2012; 2012:468609.

10. Fairman K, Motheral B. Evaluating medication adherence: Which measure is right for your program? J Manag Care Pharm 2000;6:499-504.

11. Wing RR, Koeske R, Epstein LH, et al. Long-term effects of modest weight loss in type ii diabetic patients. Arch Intern Med 1987;147:1749-1753.

12. Zillich AJ, Garg J, Basu S, et al. Thiazide diuretics, potassium, and the development of diabetes: A quantitative review. Hypertension 2006;48:219-224.

13. Van Soest EM, Siersema PD, Dieleman JP, et al. Persistence and adherence to proton pump inhibitors in daily clinical practice. Aliment Pharmacol Ther 2006;24:377385 .

14. Jimmy B, Jose J. Patient medication adherence: Measures in daily practice. Oman Med J 2011;26:155-159.

15. Turner-McGrievy GM, Jenkins DJ, Barnard ND, et al. Decreases in dietary glycemic index are related to weight loss among individuals following therapeutic diets for type 2 diabetes. J Nutr 2011;41:1469-1474.

16. ADA. Standards of medical care in diabetes 2012. Diabetes Care 2012;35:Suppl 1:S11-S63.

17. . Lazarus B, Chen Y, Wilson FP, et al. Proton pump inhibitor use and the risk of chronic kidney disease. JAMA Int Med 2016;176:238-246.

18. Little RB, Rohlfing CL, Tennill AL, et al. Measurement of HbA1c in patients with chronic renal failure. Clin Chim Acta 2013;418:73-76.

19. Takebayashi K, Inukai T. Effect of proton pump inhibitors on glycemic control in patients with diabetes. World $J$ Diabetes 2015;6:1122-1131.

20. Gómez-Izquierdo JC, Yu OHY. The Influence of protonpump inhibitors on glycemic control: A systematic review of the literature and a meta-analysis. Can J Diabetes 2017; 41:351-361.

21. Purchiaroni F, Galli G, Annibale B. Metformin plus proton pump inhibitors therapy: The cobalamin deficiency challenge. Eur Rev Med Pharmacol Sci 2015;19:2501-2502.

Address for correspondence: Arthur Swislocki, MD, FACP

Medical Service (612/111)

VA Northern California Health Care System

150 Muir Road

Martinez, CA 94553

E-mail: arthur.swislocki@va.gov 